\title{
On the Discrete Groups of Mathieu Moonshine
}

\author{
Miranda C. N. Cheng* \\ John F. R. Duncan ${ }^{\dagger}$ \\ Dedicated to Igor B. Frenkel on the occasion of his 60 th birthday.
}

\begin{abstract}
We prove that a certain space of cusp forms for the Hecke congruence group of a given level is one-dimensional if and only if that level is the order of an element of the second largest Mathieu group. As such, our result furnishes a direct analogue of Ogg's observation that the normaliser of a Hecke congruence group of prime level has genus zero if and only if that prime divides the order of the Fischer-Griess monster group. The significance of the cusp forms under consideration is explained by the Rademacher sum construction of the McKay-Thompson series of Mathieu moonshine. Our result supports a conjectural characterisation of the discrete groups and multiplier systems arising in Mathieu moonshine.
\end{abstract}

\footnotetext{
*Institut de Mathématiques de Jussieu, UMR 7586, Université Paris 7, Paris, France. E-mail: chengm@math.jussieu.fr

${ }^{\dagger}$ Department of Mathematics, Case Western Reserve University, Cleveland, OH 44106, U.S.A.

E-mail: john.duncan@case.edu
} 


\section{Contents}

1 Introduction

2 Preliminaries 6

3 Cusp forms

4 Perspectives 13

\begin{tabular}{ll} 
A Special Functions & 15 \\
\hline
\end{tabular}

\begin{tabular}{ll} 
References & 16 \\
\hline
\end{tabular}

\section{Introduction}

Ogg established one of the most surprising results relating finite groups to modular functions - a harbinger of monstrous moonshine, cf. [CN79] - by proving Ogg75 that the normalizer $\Gamma_{0}(p)^{+}$ of the Hecke congruence group

$$
\Gamma_{0}(p)=\left\{\left(\begin{array}{ll}
a & b \\
c & d
\end{array}\right) \in S L_{2}(\mathbb{Z}) \mid c \equiv 0 \quad(\bmod p)\right\}
$$

at prime level $p$ determines a genus zero quotient of the upper half-plane $\mathbb{H}$ (cf. (2.6)) if and only if $p$ is a prime dividing the order of the (at that time conjectural) Fischer-Griess monster sporadic group. Ogg offered a bottle of Jack Daniels whiskey (cf. Ogg75, Rmq.1]) to anybody who could explain this fact (and this is perhaps part of the reason the term moonshine has found the rôle that it has in mathematics).

In this article we establish an analogue of Ogg's result for Mathieu moonshine. Consider the weight $3 / 2$ cusp form given by the unary theta series

$$
\eta(\tau)^{3}=\sum_{m \in \mathbb{Z}}(4 m+1) q^{(4 m+1)^{2} / 8},
$$

which coincides with the third power of the Dedekind eta function (cf. (A.2) according to an identity due to Euler. We prove that a certain space of cusp forms for $\Gamma_{0}(p)$ with $p$ prime is one-dimensional and spanned by the above unary theta series if and only if $p$ is a prime dividing the order of the largest Mathieu sporadic group, $M_{24}$. In fact we prove more than this: the positive integers $n$ for which $\Gamma_{0}(n)$ has the above cusp form property are exactly those that arise 
as orders of elements of $M_{24}$ having a fixed point in the defining permutation representation (i.e. the unique non-trivial permutation representation on 24 points); equivalently, these values of $n$ are just those that arise as orders of elements of the second largest Mathieu sporadic group, $M_{23}$. The significance of the cusp form (1.2) and the specific space of cusp forms to be considered will soon become apparent.

Mathieu moonshine was initiated by the observation [EOT11 of Eguchi-Ooguri-Tachikawa that the low order (and non-polar) coefficients of a certain holomorphic function

$$
H(\tau)=-2 q^{-1 / 8}+90 q^{7 / 8}+462 q^{15 / 8}+1540 q^{23 / 8}+4554 q^{31 / 8}+11592 q^{39 / 8}+\ldots
$$

on the upper half-plane (we set $q=e^{2 \pi \mathbf{i} \tau}$ throughout) are simple positive-integer combinations of degrees of irreducible representations of $M_{24}$. For example, $90=45+45,462=231+$ 231, and $1540=770+770$, \&c. (cf. [CCN $\left.{ }^{+} 85\right]$ ). In [EOT11] (see also [EH09]) the function $H(\tau)$ was obtained by decomposing the elliptic genus of a $K 3$ surface into characters of the (small) $N=4$ superconformal algebra; it is essentially (up to subtraction of the polar term and multiplication by $q^{1 / 8}$ ) the generating function for multiplicities of the characters of massive (non-BPS) representations.

More significantly for the purpose of this paper, the function $H(\tau)$ is a weak mock modular form of weight $1 / 2$ for the modular group $S L_{2}(\mathbb{Z})$ with shadow equal to $24 \eta^{3}$, meaning that

$$
H(\gamma \tau) \epsilon(\gamma)^{-3} \mathrm{j}(\gamma, \tau)^{1 / 2}+\frac{e^{\pi \mathbf{i} / 4}}{2} \int_{-\gamma^{-1} \infty}^{\infty} \frac{24 \eta(z)^{3}}{\sqrt{z+\tau}} \mathrm{d} z=H(\tau)
$$

for $\tau \in \mathbb{H}$ and $\gamma \in S L_{2}(\mathbb{Z})$ and $\mathrm{j}(\gamma, \tau)=(c \tau+d)^{-1}$ in case $\gamma=\left(\begin{array}{ll}a & b \\ c & d\end{array}\right)$. In fact $H(\tau)$ is uniquely determined amongst such objects (see [CD12a, $\S 8]$ ) by having polar part $-2 q^{-1 / 8}$.

The observation relating coefficients of $H$ to degrees of irreducible representations of $M_{24}$ suggests the existence of a graded $M_{24}$-module $K=\bigoplus_{n>0} K_{n-1 / 8}$ with the property that

$$
H(\tau)=-2 q^{-1 / 8}+\sum_{n>0}\left(\operatorname{dim} K_{n-1 / 8}\right) q^{n-1 / 8} .
$$

If such a module exists then one can expect to obtain interesting functions $H_{g}(\tau)-M c K a y-$ Thompson series for $M_{24}$-by replacing $\operatorname{dim} K_{n-1 / 8}$ with $\operatorname{tr}_{K_{n-1 / 8}}(g)$ in (1.5) for $g \in M_{24}$.

$$
H_{g}(\tau)=-2 q^{-1 / 8}+\sum_{n>0} \operatorname{tr}_{K_{n-1 / 8}}(g) q^{n-1 / 8}
$$

Candidate expressions for the $H_{g}$ were determined in a series of papers, starting with Che10. 
and the independent work GHV10b], and concluding with GHV10a] and EH11]. A proof of the existence of the $M_{24}$-module $K$, with McKay-Thompson series given exactly as predicted in Che10, GHV10b, GHV10a, EH11, has appeared very recently Gan12; as yet no concrete construction of $K$ is known.

It develops that the McKay-Thompson series all enjoy good modular properties. To describe them we set $n_{g}$ to be the order of $g$, we write $\chi(g)$ for the number of fixed points of $g$ in the defining permutation representation and define $h_{g}$ to be the length of a minimal length cycle in a disjoint cycle decomposition of (the permutation induced by) $g$ (cf. CD12a, CD12b). For instance, for an element $g$ in the (unique) conjugacy class with cycle shape $1^{8} 2^{8}$ we have $n_{g}=2$, $h_{g}=1$ and $\chi(g)=8$; for an element $g$ in the (unique) conjugacy class with cycle shape $2^{12}$ we have $n_{g}=h_{g}=2$ and $\chi(g)=0$. In particular, $h_{g}=1$ and $\chi(g) \neq 0$ just when $g$ has a fixed point. By definition $h_{g}$ divides $n_{g}$ and by inspection $h_{g}$ also divides 12 for each $g \in M_{24}$. In this way we attach to each element $g \in M_{24}$ a discrete group $\Gamma_{g}=\Gamma_{0}\left(n_{g}\right)<S L_{2}(\mathbb{Z})$ and a multiplier system $\psi_{g}=\rho_{n \mid h} \epsilon^{-3}$ with weight $1 / 2$, where $n=n_{g}$ and $h=h_{g}$. Here $\epsilon$ denotes the multiplier system of the Dedekind eta function (cf. A.3. $)$. The function $\rho_{n \mid h}: \Gamma_{0}(n) \rightarrow \mathbb{C}^{\times}$is defined for any pair of positive integers $n$ and $h$ such that $h$ divides $\operatorname{gcd}(n, 12)$ by setting

$$
\rho_{n \mid h}\left(\begin{array}{ll}
a & b \\
c & d
\end{array}\right)=\exp \left(-2 \pi \mathbf{i} \frac{c d}{n h}\right)
$$

Since $h$ is a divisor of 24 we have $x \equiv y(\bmod h)$ whenever $x y \equiv 1(\bmod h)(\mathrm{cf}$. CN79, §3]) and it follows from this that $\rho_{n \mid h}$ is actually a morphism of groups; the kernel is evidently $\Gamma_{0}(n h)$. Now we may describe the modularity of all the McKay-Thompson series $H_{g}$ by stating that for arbitrary $g \in M_{24}$ the function $H_{g}$ is a weak mock modular form for $\Gamma_{0}\left(n_{g}\right)$ with weight $1 / 2$ and multiplier $\rho_{n \mid h} \epsilon^{-3}$, where $n=n_{g}$ and $h=h_{g}$, and the shadow of $H_{g}$ is $\chi(g) \eta^{3}$. See (2.5) for the definition of weak mock modular form. Notice that the functions $H_{g}$ for $g \in M_{24}$ with and without fixed points have qualitatively different modular behaviour: the former are weak mock modular forms on $\Gamma_{0}\left(n_{g}\right)$ with non-trivial shadow while the latter are actually weak modular forms.

In CD12a it is shown that a uniform construction of the McKay-Thompson series $H_{g}$ may be obtained using Rademacher sums. The analogous statement for monstrous moonshine was established in [DF11, while in CDH12] it was conjectured that an analogous Rademacher sum construction also exists for the McKay-Thompson series of umbral moonshine. The fact that the McKay-Thompson series in these various settings may all be constructed using Rademacher sums indicates a deep relation between Rademacher sums and moonshine. 
To explore this relation further, and to motivate the spaces of cusp forms we study in this work, recall (from [CD12c], for example) that given a group $\Gamma<S L_{2}(\mathbb{R})$ commensurable with $S L_{2}(\mathbb{Z})$, a multiplier system $\psi$ for $\Gamma$, a compatible weight $w$ and a compatible index $\mu$, we may consider the Rademacher sum $R_{\Gamma, \psi, w}^{[\mu]}$ (we refer to $[\mathrm{CD} 12 \mathrm{c}$ for the definition). In the case that it converges $R_{\Gamma, \psi, w}^{[\mu]}$ defines a weak mock modular form for the group $\Gamma$ with multiplier $\psi$ and weight $w$, and Fourier expansion of the form $R_{\Gamma, \psi, w}^{[\mu]}(\tau)=q^{\mu}+O(1)$. (We typically have $w<1$ and $\mu<0$ in applications.) The shadow of $R_{\Gamma, \psi, w}^{[\mu]}$ is a modular form for $\Gamma$ - a cusp form in case $\mu<0$ - with the inverse multiplier $\bar{\psi}$ and dual weight $2-w$, and is itself a Rademacher sum. We refer to $[\mathrm{CD12 \textrm {C }}$ for an exposition.

Write $S_{\psi, w}(\Gamma)$ for the space of cusp forms for $\Gamma$ with multiplier $\psi$ and weight $w$, so that $S_{\psi, w}(\Gamma)$ is the space of possibilities for the shadow of a Rademacher sum $R_{\Gamma, \bar{\psi}, 2-w}^{[\mu]}$. As we shall see presently the Rademacher sums of monstrous and Mathieu moonshine have the common feature that their corresponding spaces $S_{\psi, w}(\Gamma)$ are extremely small; viz., zero or one dimensional, and this feature plays an important rôle in determining the Rademacher sums themselves. From this point of view Ogg's result and the result of the present paper may be viewed as two manifestations of a single (as yet empirical) principle of the Rademacher sum construction.

In the case of monstrous moonshine the relevant Rademacher sums have trivial multiplier, weight 0 and $\mu=-1$ [DF11] and thus their shadows lie in the spaces $S_{2}(\Gamma)=S_{1,2}(\Gamma)$ of cusp forms with trivial multiplier of weight 2 . The fact that the dimension of $S_{2}(\Gamma)$ coincides with the genus of the compact Riemann surface $X_{\Gamma}$ determined by $\Gamma$ (cf. (2.6) ) indicates the close connection between Rademacher sums and the genus zero property: the weight 0 Rademacher $\operatorname{sum} R_{\Gamma, 1,0}^{[-1]}$ is forced to have trivial shadow, and thus be $\Gamma$-invariant (i.e. a weight 0 weak modular form for $\Gamma$ ), whenever $X_{\Gamma}$ has genus zero since in that case the space of possible shadows $S_{2}(\Gamma)$ is zero-dimensional. In DF11] it is shown that the converse is also true, so that a discrete group $\Gamma$ determines a genus zero surface $X_{\Gamma}$ if and only if the Rademacher sum $R_{\Gamma, 1,0}^{[-1]}$ is $\Gamma$-invariant, and the genus zero property of monstrous moonshine is thus reformulated in terms of Rademacher sums. (As such, the main result of CD12a may be regarded as verifing a natural analogue of the genus zero property for Mathieu moonshine; we refer to [CD12b, CD12c for a fuller discussion.) In particular, the equality of a monstrous McKay-Thompson series $T_{g}$ with $R_{\Gamma_{g}, 1,0}^{[-1]}$ (up to an additive constant, cf. [DF11, CD12C) implies that $\Gamma_{g}$ has genus zero.

To recover the McKay-Thompson series of Mathieu moonshine corresponding to elements with fixed points we choose a positive integer $n$ and set $\Gamma=\Gamma_{0}(n)$ and $\psi=\epsilon^{-3}$ (cf. A.30) and $w=1 / 2$ and also $\mu=-1 / 8$. Then the space of possible shadows for the Rademacher sum $R_{\Gamma_{0}(n), \epsilon^{-3}, 1 / 2}^{[-1 / 8]}$ is $S_{\epsilon^{3}, 3 / 2}\left(\Gamma_{0}(n)\right)$, and we abbreviate this to $S_{\epsilon^{3}, 3 / 2}(n)$ to ease notation. We can see immediately that $S_{\epsilon^{3}, 3 / 2}(n)$ has positive dimension for all positive $n$ since the cusp form 
$\eta^{3}$ (cf. (1.2)) is a non-zero element of $S_{\epsilon^{3}, 3 / 2}(1)$, and $S_{\epsilon^{3}, 3 / 2}(m)$ embeds in $S_{\epsilon^{3}, 3 / 2}(n)$ whenever $m \mid n$. In the course of proving [CD12a] that the McKay-Thompson series $H_{g}$ determined in the aforementioned articles Che10, GHV10b, GHV10a, EH11] satisfy $H_{g}=-2 R_{\Gamma_{0}(n), \epsilon^{-3,1 / 2}}^{[-1 / 8]}$ for $n=n_{g}$ when $g$ has a fixed point, it was shown that $S_{\epsilon^{3}, 3 / 2}(n)$ is one-dimensional, spanned by $\eta^{3}$, whenever $n$ is the order of an element of $M_{23}$ (cf. [CD12a, §8.1]). In particular, the restricted nature of the space $S_{\epsilon^{3}, 3 / 2}(n)$ was crucial for the purposes of determining the Rademacher sums associated to elements of the subgroup $M_{23}$.

In this article we make an important step towards a characterisation of the discrete groups and multipliers of Mathieu moonshine, and in so doing strengthen the analogy with the monstrous case just described, by proving the converse statement. We identify an isomorphism between $S_{\epsilon^{3}, 3 / 2}(n)$ and a certain space of meromorphic functions on the modular curve $X_{0}(n)$ (cf. (2.6|2.7) ) and use this together with the Riemann-Roch theorem to prove that $\operatorname{dim} S_{\epsilon^{3}, 3 / 2}(n)=1$ if and only if $n$ is the order of an element of $M_{23}$. Since there is an element in $M_{23}$ with order $p$ for any prime $p$ dividing the order of $M_{24}$ we obtain a direct analogue of Ogg's result characterising the primes $p$ for which $\Gamma_{0}(p)^{+}$has genus zero in terms of the Monster group: the primes $p$ for which $\Gamma_{0}(p)$ has a unique (up to scale) cusp form of weight $3 / 2$ with multiplier $\epsilon^{3}$ are exactly those that divide the order of $M_{24}$, which are also just the primes $p$ such that $p+1$ divides 24 .

The rest of the paper is organised as follows. In $\oint 2$ we collect preliminary definitions and notations. In $\$ 3$ we present the proof of the main theorem of the paper. We conclude in 4 with some perspectives on possible future developments, including a conjectural characterisation of the discrete groups and multipliers of Mathieu moonshine.

\section{Preliminaries}

The group $S L_{2}(\mathbb{R})$ acts naturally on the upper half-plane $\mathbb{H}$ by orientation preserving isometries according to the rule

$$
\left(\begin{array}{ll}
a & b \\
c & d
\end{array}\right) \tau=\frac{a \tau+b}{c \tau+d} .
$$

For $\gamma \in S L_{2}(\mathbb{R})$ with lower row $(c, d)$ define

$$
\mathrm{j}(\gamma, \tau)=(c \tau+d)^{-1}
$$


so that $\mathrm{j}(\gamma, \tau)^{2}$ is the derivative (with respect to $\tau$ ) of the action (2.1) when $\gamma=\left(\begin{array}{ll}a & b \\ c & d\end{array}\right)$. For $\Gamma$ a finite index subgroup of the modular group $S L_{2}(\mathbb{Z})$ and for $w \in \mathbb{R}$ say that a function $\psi: \Gamma \rightarrow \mathbb{C}$ is a multiplier system for $\Gamma$ with weight $w$ if

$$
\psi\left(\gamma_{1}\right) \psi\left(\gamma_{2}\right) \mathrm{j}\left(\gamma_{1}, \gamma_{2} \tau\right)^{w} \mathrm{j}\left(\gamma_{2}, \tau\right)^{w}=\psi\left(\gamma_{1} \gamma_{2}\right) \mathrm{j}\left(\gamma_{1} \gamma_{2}, \tau\right)^{w}
$$

for all $\gamma_{1}, \gamma_{2} \in \Gamma$ where here and everywhere else in this paper we choose the principal branch of the logarithm (cf. (A.1)) in order to define the exponential $x \mapsto x^{s}$ in case $s$ is not an integer. Given a multiplier system $\psi$ for $\Gamma$ with weight $w$ we may define the $(\psi, w)$-action of $\Gamma$ on the space $\mathcal{O}(\mathbb{H})$ of holomorphic functions on the upper half-plane by setting

$$
\left(\left.f\right|_{\psi, w} \gamma\right)(\tau)=f(\gamma \tau) \psi(\gamma) \mathrm{j}(\gamma, \tau)^{w}
$$

for $f \in \mathcal{O}(\mathbb{H})$ and $\gamma \in \Gamma$. We then say that $f \in \mathcal{O}(\mathbb{H})$ is an unrestricted modular form with multiplier $\psi$ and weight $w$ for $\Gamma$ in the case that $f$ is invariant for this action; i.e. $\left.f\right|_{\psi, w} \gamma=f$ for all $\gamma \in \Gamma$. Since $(-\gamma) \tau=\gamma \tau$ and $\mathrm{j}(-\mathrm{Id}, \tau)^{w}=e^{-\pi \mathrm{i} w}$ (cf. A.1) the multiplier $\psi$ must satisfy the consistency condition $\psi(-\mathrm{Id})=e^{\pi \mathbf{i} w}$ in order that the corresponding space(s) of unrestricted modular forms be non-vanishing when $-\mathrm{Id} \in \Gamma$.

We assume throughout that the multiplier $\psi$ for $\Gamma$ is of the form $\psi=\rho \tilde{\psi}$ where $\rho: \Gamma \rightarrow \mathbb{C}^{\times}$ is a morphism of groups and $\tilde{\psi}$ is a multiplier for $S L_{2}(\mathbb{Z})$. With this understanding we say that an unrestricted modular form $f$ for $\Gamma$ with multiplier $\psi$ and weight $w$ is a weak modular form in case $f$ has at most exponential growth at the cusps of $\Gamma$; i.e. in case there exists $C>0$ such that $\left(\left.f\right|_{\tilde{\psi}, w} \sigma\right)(\tau)=O\left(e^{C \Im(\tau)}\right)$ as $\Im(\tau) \rightarrow \infty$ for any $\sigma \in S L_{2}(\mathbb{Z})$. We say that $f$ is a modular form if $\left(\left.f\right|_{\tilde{\psi}, w} \sigma\right)(\tau)$ remains bounded as $\Im(\tau) \rightarrow \infty$ for any $\sigma \in S L_{2}(\mathbb{Z})$, and we say $f$ is a cusp form if $\left(\left.f\right|_{\tilde{\psi}, w} \sigma\right)(\tau) \rightarrow 0$ as $\Im(\tau) \rightarrow \infty$ for any $\sigma \in S L_{2}(\mathbb{Z})$.

Suppose that $\psi$ is a multiplier system for $\Gamma$ with weight $w$ and $g$ is a modular form for $\Gamma$ with the inverse multiplier system $\bar{\psi}: \gamma \mapsto \overline{\psi(\gamma)}$ and $d u a l$ weight $2-w$. Then we may use $g$ to twist the $(\psi, w)$-action of $\Gamma$ on $\mathcal{O}(\mathbb{H})$ by setting

$$
\left(\left.f\right|_{\psi, w, g} \gamma\right)(\tau)=f(\gamma \tau) \psi(\gamma) \mathrm{j}(\gamma, \tau)^{w}+\left(\frac{\mathbf{i}}{4}\right)^{1-w} \int_{-\gamma^{-1} \infty}^{\infty}(z+\tau)^{-w} \overline{g(-\bar{z})} \mathrm{d} z .
$$

A weak mock modular form for $\Gamma$ with multiplier $\psi$, weight $w$, and shadow $g$ is a holomorphic function $f$ on $\mathbb{H}$ that is invariant for the $(\psi, w, g)$-action of $\Gamma$ defined in (2.5) and which has at most exponential growth at the cusps of $\Gamma$ (i.e. there exists $C>0$ such that $\left(\left.f\right|_{\tilde{\psi}, w} \sigma\right)=$ $O\left(e^{C \Im(\tau)}\right)$ for all $\sigma \in S L_{2}(\mathbb{Z})$ as $\Im(\tau) \rightarrow \infty$ where $\tilde{\psi}$ is as in the previous paragraph.) A weak 
mock modular form is called a mock modular form in case it is bounded at every cusp. From this point of view a (weak) modular form is a (weak) mock modular form with vanishing shadow. The notion of mock modular form developed from Zwegers' ground breaking work [Zwe02] on Ramanujan's mock theta functions. We refer to Zag09 for an excellent review. The notion of mock modular form is closely related to the notion of automorphic integral which was introduced by Niebur in Nie74]; see [CD12C for a discussion of this.

Since $\Gamma$ is assumed to be a subgroup of $S L_{2}(\mathbb{Z})$ of finite index its natural action on the boundary $\hat{\mathbb{R}}=\mathbb{R} \cup\{\infty\}$ of $\mathbb{H}$ restricts to $\hat{\mathbb{Q}}=\mathbb{Q} \cup\{\infty\}$. The orbits of $\Gamma$ on $\hat{\mathbb{Q}}$ are called the cusps of $\Gamma$. The quotient space

$$
X_{\Gamma}=\Gamma \backslash \mathbb{H} \cup \hat{\mathbb{Q}}
$$

is naturally a compact Riemann surface (cf. e.g. [Shi71, §1.5]). We say that $\Gamma$ has genus $g$ in case $X_{\Gamma}$ has genus $g$ as an orientable surface.

In this paper we will be concerned primarily with the case that $\Gamma$ is the Hecke congruence group of level $n$, denoted $\Gamma_{0}(n)$, for some integer $n$.

$$
\Gamma_{0}(n)=\left\{\left(\begin{array}{ll}
a & b \\
c & d
\end{array}\right) \in S L_{2}(\mathbb{Z}) \mid c \equiv 0 \quad(\bmod n)\right\}
$$

We write $X_{0}(n)$ for $X_{\Gamma}$ when $\Gamma=\Gamma_{0}(n)$.

\section{Cusp forms}

Recall that $S_{\epsilon^{3}, 3 / 2}(n)$ denotes the space of cusp forms for $\Gamma_{0}(n)$ with multiplier $\epsilon^{3}$ and weight $3 / 2$. Then the function $\eta^{3}$ belongs to $S_{\epsilon^{3}, 3 / 2}(n)$ for every positive integer $n$, so in particular $S_{\epsilon^{3}, 3 / 2}(n)$ has dimension at least 1 for all $n$. We will prove that the values of $n$ for which $\operatorname{dim} S_{\epsilon^{3}, 3 / 2}(n)=1$ are exactly those that arise as the order of an element of the sporadic group $M_{23}$; viz., $n \in\{1,2,3,4,5,6,7,8,11,14,15,23\}$.

Observe that if $g \in S_{\epsilon^{3}, 3 / 2}(n)$ then $\tilde{g}=g \eta^{-3}$ is a meromorphic function on $X=X_{0}(n)$ with poles only at the cusps of $\Gamma=\Gamma_{0}(n)$, and the order of the pole at a cusp $x \in \Gamma \backslash \hat{\mathbb{Q}}$ say is bounded from above by the order of vanishing of $\eta^{3}$ at $x$. For a more precise statement define a divisor $D$ on $X$ by setting

$$
D=\sum_{x \in \Gamma \backslash \hat{\mathbb{Q}}}\left(\left\lceil\frac{w_{x}}{8}\right\rceil-1\right) x
$$


where $w_{x}$ denotes the width of $\Gamma$ at the cusp $x$. Write $\mathcal{K}_{X}(D)$ for the vector space composed of meromorphic functions $f$ on $X$ satisfying $(f)+D \geq 0$. Then $g \eta^{-3}$ belongs to $\mathcal{K}_{X}(D)$ whenever $g \in S_{\epsilon^{3}, 3 / 2}(n)$, and conversely, if $\tilde{g} \in \mathcal{K}_{X}(D)$ then $\tilde{g} \eta^{3}$ belongs to $S_{\epsilon^{3}, 3 / 2}(n)$. So multiplication by $\eta^{3}$ defines an isomorphism of vector spaces $\mathcal{K}_{X}(D) \rightarrow S_{\epsilon^{3}, 3 / 2}(n)$. According to the RiemannRoch Theorem we have $\operatorname{dim} \mathcal{K}_{X}(D)-\operatorname{dim} \Omega_{X}(D)=\operatorname{deg}(D)+1-\operatorname{genus}(X)$ where $\Omega_{X}(D)$ denotes the space of holomorphic differentials $\omega$ on $X$ satisfying $(\omega)-D \geq 0$. In particular then

$$
S_{\epsilon^{3}, 3 / 2}(n) \geq \operatorname{deg}(D)+1-\operatorname{genus}(X)
$$

when $D$ is given by (3.1) and $X=X_{0}(n)$. In the case that $X=X_{0}(n)$ we have the explicit formula (cf. [Ste07, §6.1])

$$
\operatorname{genus}\left(X_{0}(n)\right)=1+\frac{1}{12} i(n)-\frac{1}{4} \mu_{2}(n)-\frac{1}{3} \mu_{3}(n)-\frac{1}{2} c(n)
$$

where $i(n)$ is the index of $\Gamma_{0}(n)$ in the modular group $S L_{2}(\mathbb{Z})$, and $c(n)=\# \Gamma_{0}(n) \backslash \hat{\mathbb{Q}}$ is the number of cusps of $\Gamma_{0}(n)$, and $\mu_{2}(n)$ and $\mu_{3}(n)$ are defined by setting

$$
\begin{gathered}
\mu_{2}(n)= \begin{cases}0 & \text { if } 4 \mid n, \\
\prod_{p \mid n}\left(1+\left(\frac{-4}{p}\right)\right) & \text { otherwise }\end{cases} \\
\mu_{3}(n)= \begin{cases}0 & \text { if } 2 \mid n \text { or } 9 \mid n, \\
\prod_{p \mid n}\left(1+\left(\frac{-3}{p}\right)\right) & \text { otherwise },\end{cases}
\end{gathered}
$$

where $\left(\frac{k}{p}\right)$ denotes the Kronecker symbol (cf. 3 A ). Substituting (3.3) and the expression (3.1) for $D$ into (3.2) we thus obtain the lower bound

$$
\operatorname{dim} S_{\epsilon^{3}, 3 / 2}(n) \geq \sum_{x \in \Gamma \backslash \hat{\mathbb{Q}}}\left\lceil\frac{w_{x}}{8}\right\rceil-\frac{1}{12} i(n)-\frac{1}{2} c(n)+\frac{1}{4} \mu_{2}(n)+\frac{1}{3} \mu_{3}(n)
$$

on $\operatorname{dim} S_{\epsilon^{3}, 3 / 2}(n)$. A weaker but still useful lower bound is the formula

$$
\operatorname{dim} S_{\epsilon^{3}, 3 / 2}(n) \geq \sum_{x \in \Gamma \backslash \hat{\mathbb{Q}}}\left\lceil\frac{w_{x}}{8}\right\rceil-\frac{1}{12} i(n)-\frac{1}{2} c(n),
$$

obtained by ignoring the contribution to genus $\left(X_{0}(n)\right)$ of elliptic points on $X_{0}(n)$. Since $i(n)$ coincides with the sum of the widths of the cusps of $\Gamma_{0}(n)$ (cf. e.g. [DF11, §5.3]) we have the 
crude lower bound

$$
\operatorname{dim} S_{\epsilon^{3}, 3 / 2}(n) \geq \frac{1}{24} i(n)-\frac{1}{2} c(n)
$$

which shows at a glance that $S_{\epsilon^{3}, 3 / 2}(n)$ has dimension greater than 1 for sufficiently large $n$ since the index of $\Gamma_{0}(n)$ in the modular group grows faster with $n$ than does the number of cusps of $\Gamma_{0}(n)$. Precise formulas for $i(n)$ and $c(n)$ are as follows (cf. e.g. [Ste07, §6.1]).

$$
\begin{aligned}
& i(n)=\prod_{p \mid n}\left(p^{\nu_{p}(n)}+p^{\nu_{p}(n)-1}\right) \\
& c(n)=\sum_{d \mid n} \phi(\operatorname{gcd}(d, n / d))
\end{aligned}
$$

In (3.9) we write $\nu_{p}(n)$ for the greatest positive integer such that $p^{\nu_{p}(n)}$ divides $n$. In (3.10) we write $\phi$ for the Euler totient function.

The cusps of $\Gamma_{0}(n)$ are indexed by equivalence classes of pairs $(a, d)$ where $d \mid n$ and $\operatorname{gcd}(a, d)=$ 1 , and $(a, d) \sim\left(a^{\prime}, d^{\prime}\right)$ just when $d=d^{\prime}$ and $a \equiv a^{\prime} \operatorname{modulo} \operatorname{gcd}(d, n / d)$. Writing $d=\prod_{p \mid n} p^{\nu_{p}(d)}$, the width of the cusp corresponding to a pair $(a, d)$ is given by

$$
\prod_{p \mid n} p^{\left(\nu_{p}(n)-2 \nu_{p}(d)\right) H\left[\nu_{p}(n)-2 \nu_{p}(d)\right]}
$$

where $H[n]$ is the Heaviside step function given by 0 when $n<0$ and 1 when $n \geq 0$.

Using (3.6) and (3.7) together with (3.9) and (3.10) we will now show that $\operatorname{dim} S_{\epsilon^{3}, 3 / 2}(n)>1$ for all $n$ except those that arise as the order of an element of the Mathieu group $M_{23}$. We start with the following result on prime powers.

Lemma 3.1. If $n$ is a prime power then $\operatorname{dim} S_{\epsilon^{3}, 3 / 2}(n)>1$ for $n \notin\{1,2,3,5,7,11,23,4,8\}$.

Proof. First consider the case that $n=p$ is prime. We have $i(p)=p+1$ and $c(p)=2$. The cusp at infinity has width 1 and the cusp represented by $0 \in \hat{\mathbb{Q}}$ has width $p \operatorname{so} \operatorname{dim} S_{\epsilon^{3}, 3 / 2}(p) \geq$ $(p-2) / 24$ according to (3.7) and thus $\operatorname{dim}_{\epsilon^{3}, 3 / 2}(n)>1$ when $n$ is a prime greater than 23 . For the case that $n$ is prime it remains to show that $\operatorname{dim} S_{\epsilon^{3}, 3 / 2}(p)>1$ for $p \in\{13,17,19\}$ and this follows from (3.6) together with the explicit computations $\mu_{2}(13)=\mu_{2}(17)=\mu_{3}(13)=\mu_{3}(19)=2$ and $\mu_{2}(19)=\mu_{3}(17)=0$.

Now suppose that $n=p^{2 \lambda}$ for some $\lambda \geq 1$. Then we have $i\left(p^{2 \lambda}\right)=p^{2 \lambda}+p^{2 \lambda-1}$ and $c\left(p^{2 \lambda}\right)=p^{\lambda}+p^{\lambda-1}$, and there are $p^{\lambda}$ cusps of width 1 , one cusp of width $p^{2 \lambda}$, and $p^{\lambda-k}-p^{\lambda-k-1}$ cusps of width $p^{2 k}$ for every $k=1, \ldots, \lambda-1$. To get a simple bound on $\operatorname{dim} S_{\epsilon^{3}, 3 / 2}\left(p^{2 \lambda}\right)$ we use 
the fact that $p^{\lambda}$ cusps have width 1 and obtain the lower bound

$$
\sum_{x \in \Gamma \backslash \hat{\mathbb{Q}}}\left\lceil\frac{w_{x}}{8}\right\rceil \geq \frac{p^{2 \lambda}+p^{2 \lambda-1}-p^{\lambda}}{8}+p^{\lambda},
$$

and then an application of (3.7) yields

$$
\operatorname{dim} S_{\epsilon^{3}, 3 / 2}\left(p^{2 \lambda}\right) \geq \frac{p^{2 \lambda}+p^{2 \lambda-1}+9 p^{\lambda}-12 p^{\lambda-1}}{24} .
$$

Taking $\lambda=1$ in (3.13) we see that $\operatorname{dim} S_{\epsilon^{3}, 3 / 2}(n)>1$ whenever $n$ is the square of a prime greater than 2, and taking $\lambda=2$ in (3.13) we see that $\operatorname{dim} S_{\epsilon^{3}, 3 / 2}(n)>1$ whenever $n$ is the fourth power of any prime. Using now the elementary fact that $S_{\epsilon^{3}, 3 / 2}(m)$ is naturally identified with a subspace of $S_{\epsilon^{3}, 3 / 2}(n)$ whenever $m$ is a divisor of $n$ we conclude that $\operatorname{dim} S_{\epsilon^{3}, 3 / 2} \geq 2$ whenever $n$ is a prime power not in the set $\{1,2,3,5,7,11,23,4,8\}$, as we required to show.

Lemma 3.2. If $n=\prod_{i=1}^{k} p_{i}$ is a square-free product of distinct primes, then $\operatorname{dim} S_{\epsilon^{3}, 3 / 2}(n)>1$ unless $n \in\{6,14,15\}$.

Proof. For $n=\prod_{i=1}^{k} p_{i}$ we have $i(n)=\prod_{i=1}^{k}\left(p_{i}+1\right)$ and $c(n)=2^{k}$. Hence the crude estimate (3.8) gives

$$
\operatorname{dim} S_{\epsilon^{3}, 3 / 2}(n)>\frac{1}{24} \prod_{i=1}^{k}\left(p_{i}+1\right)-2^{k-1} .
$$

Take the case $k=2$ and $n=p q$. Then the above formula shows that $\operatorname{dim} S_{\epsilon^{3}, 3 / 2}(n) \geq 2$ if both $p$ and $q$ are larger than 8. Without loss of generality let $p<q$. To refine (3.14) we put $\left\lceil\frac{p}{8}\right\rceil=1$ in (3.7) and obtain

$$
\operatorname{dim} S_{\epsilon^{3}, 3 / 2}(p q)>\frac{p q}{8}+\frac{q}{8}-\frac{1}{12}(p+1)(q+1)=\frac{1}{24}(p+1)(q-2)
$$

and thus $\operatorname{dim} S_{\epsilon^{3}, 3 / 2}(p q)>1$ for $p q$ not in the set $\{6,10,14,15,21\}$.

For $k=3$ the crude estimate (3.14) excludes all possibilities except for $n=30$ and $n=42$. Similarly, it excludes all possibilities with $k>3$, and so it remains to show that $\operatorname{dim} S_{\epsilon^{3}, 3 / 2}(n)>1$ for $n \in\{10,21,30,42\}$. This follows in each case from an explicit computation of $\mu_{2}(n)$ and $\mu_{3}(n)$ and an application of (3.6).

Lemma 3.3. We have $\operatorname{dim} S_{\epsilon^{3}, 3 / 2}(n)>1$ whenever $n>1$ is not a prime power and is not square-free. 
Proof. From the fact, mentioned above, that $\operatorname{dim} S_{\epsilon^{3}, 3 / 2}(n) \geq S_{\epsilon^{3}, 3 / 2}(m)$ whenever $m$ divides $n$ we conclude from Lemma 3.1 that the statement is automatically true unless $n=4 p$ or $n=8 p$ with $p \in\{3,5,7,11,23\}$.

The group $\Gamma_{0}(4 p)$ has two cusps of width 1 , two cusps of width $p$, one cusp of width 4 and one with width $4 p$. This gives $\operatorname{deg}(D)>\frac{3 p}{4}-3$. On the other hand, we have $i(4 p)=6 p+6$ and $c(4 p)=6$ and this gives $\operatorname{genus}(X) \leq \frac{p-3}{2}$, and so we obtain $\operatorname{dim} S_{\epsilon^{3}, 3 / 2}(4 p) \geq \frac{p-2}{4}$ from (3.2), and hence $\operatorname{dim} S_{\epsilon^{3}, 3 / 2}(4 p)>1$ unless $p=3$ or $p=5$. For $n=12$ we compute $\operatorname{deg} D=1$ and $\operatorname{genus}(X)=0$, and this gives $\operatorname{dim} S_{\epsilon^{3}, 3 / 2}(12)>1$ via (3.2). Similarly for $n=20$ we have $\operatorname{deg} D=2$ and $\operatorname{genus}(X)=1$ and so $\operatorname{dim} S_{\epsilon^{3}, 3 / 2}(20)>1$ also. Therefore $\operatorname{dim} S_{\epsilon^{3}, 3 / 2}(4 p)>1$ for all $p>2$. Then it follows that $\operatorname{dim} S_{\epsilon^{3}, 3 / 2}(8 p) \geq \operatorname{dim} S_{\epsilon^{3}, 3 / 2}(4 p)>1$ for all $p>2$ and this finishes the proof.

For the sake of completeness we conclude with a proof of the result, established earlier in CD12a, that $\operatorname{dim} S_{\epsilon^{3}, 3 / 2}(n)=1$ whenever $n$ is the order of an element of $M_{23}$.

Lemma 3.4. If $n \in\{1,2,3,4,5,6,7,8,11,14,15,23\}$ then $\operatorname{dim} S_{\epsilon^{3}, 3 / 2}(n)=1$.

Proof. For $n \leq 8$ we have $D=0$. Since every holomorphic function on a compact Riemann surface is a constant we have $\mathcal{K}_{X}(D)=\mathbb{C}$ and as a result $\operatorname{dim} S_{\epsilon^{3}, 3 / 2}(n)=\operatorname{dim} \mathcal{K}_{X}(D)=1$. For $n \in\{11,14,15\}$ there is just one cusp of $\Gamma_{0}(n)$ (the one represented by 1 ) that has width larger than 8. In these cases, denote by $x$ the image of $1 \in \hat{\mathbb{Q}}$ under the natural map $\mathbb{H} \cup \hat{\mathbb{Q}} \rightarrow X_{0}(n)$, then any $f \in \mathcal{K}_{X}(D)$ is either a constant or has a simple pole at $x$ and no other poles, but the latter is impossible since such an $f$ would induce an isomorphism between the Riemann sphere and $X_{0}(n)$, which is a genus one curve for $n \in\{11,14,15\}$. As a result we conclude $\mathcal{K}_{X}(D)=\mathbb{C}$ and $\operatorname{dim} S_{\epsilon^{3}, 3 / 2}(n)=\operatorname{dim} \mathcal{K}_{X}(D)=1$.

It remains to show that $\operatorname{dim} S_{\epsilon^{3}, 3 / 2}(23)=1$. The group $\Gamma_{0}(23)$ has two cusps, one of width 23 represented by 1 , and one with width 1 represented by $1 / 23$. As a result we have $D=2 x$ where $x$ again denotes the image of $1 \in \hat{\mathbb{Q}}$ under $\mathbb{H} \cup \hat{\mathbb{Q}} \rightarrow X_{0}(23)$. Consider first $D^{\prime}=x$. Repeating the above argument and using the fact that $X_{0}(23)$ has genus 2 we conclude that $\operatorname{dim} \Omega_{X}\left(D^{\prime}\right)=\operatorname{dim} \mathcal{K}_{X}\left(D^{\prime}\right)=1$. From the fact that $\Omega_{X}\left(D^{\prime}\right) \supset \Omega_{X}(D)$ and the one dimensional space $\Omega_{X}\left(D^{\prime}\right)$ is given by the weight 2 cusp form $\eta(\tau)^{2} \eta(23 \tau)^{2}$, we conclude that $\operatorname{dim} \Omega_{X}(D)=0$ and hence $\operatorname{dim} S_{\epsilon^{3}, 3 / 2}(23)=1$.

Taking the above lemmas together we obtain our main result.

Theorem 3.5. We have $\operatorname{dim} S_{\epsilon^{3}, 3 / 2}(n)=1$ if and only if $n$ coincides with the order of an element of the sporadic group $M_{23}$; viz., $n \in\{1,2,3,4,5,6,7,8,11,14,15,23\}$. 


\section{Perspectives}

As has been explained in the introduction, the fact that the space $S_{\epsilon^{3}, 3 / 2}(n)$ has dimension 1 whenever $n$ is the order of an element of $M_{23}$ may, in light of the Rademacher sum construction of the McKay-Thompson series of Mathieu moonshine, be regarded as a counterpart to the genus zero property of monstrous moonshine since the latter is equivalent to the existence of Rademacher sum expressions for the monstrous McKay-Thompson series. This equivalence was extended in [DF11] so as to obtain a Rademacher sum-based characterisation of the groups $\Gamma_{g}<$ $S L_{2}(\mathbb{R})$ for $g$ in the monster by reformulating an earlier characterisation due to Conway-McKaySebbar [CMS04]. The main result of this article, determining those $n$ for which $\operatorname{dim} S_{\epsilon^{3}, 3 / 2}(n)=$ 1 , serves as evidence in support of an analogous characterisation of the discrete groups (and multipliers) of Mathieu moonshine.

As in $\$ 1$ we attach a discrete group $\Gamma_{g}=\Gamma_{0}(n)<S L_{2}(\mathbb{Z})$ and a multiplier system $\psi_{g}=$ $\rho_{n \mid h} \epsilon^{-3}$ (cf. 1.7) ) with weight $1 / 2$ to each element $g \in M_{24}$. Following the suggestion made in CD12c we conjecture that the pairs $(\Gamma, \psi)$ arising as $\left(\Gamma_{g}, \psi_{g}\right)$ for $g \in M_{24}$ are exactly those of the form $(\Gamma, \psi)=\left(\Gamma_{0}(n), \rho_{n \mid h} \epsilon^{-3}\right)$ where

1. $n$ and $h$ are positive integers such that $h$ divides $\operatorname{gcd}(n, 12)$, and

2. the Rademacher sum $R_{\Gamma, \psi, 1 / 2}^{[-1 / 8]}$ has shadow proportional to $\eta^{3}$.

The main result of this article implies that if $h=1$ then the second condition above is necessarily satisfied when $n$ is the order of an element of $M_{23}$. It also suggests that the second condition is unlikely to be satisfied when $h=1$ and $n$ is not the order of an element of $M_{23}$, for then, according to our result, the space of possible shadow functions has dimension greater than one.

Observe that the second condition actually implies that $R_{\Gamma, \psi, 1 / 2}^{[-1 / 8]}$ has vanishing shadow - and is thus a weak modular form - when $h>1$, for the multiplier of $\eta^{3}$ agrees with $\overline{\rho_{n \mid h} \epsilon^{-3}}$ on $\Gamma_{0}(n h)$ but differs from it on non-trivial cosets of $\Gamma_{0}(n h)$ in $\Gamma_{0}(n)$. A next step towards establishing the above conjectural characterization of the discrete groups and multipliers of Mathieu moonshine would be to carry out the $h>1$ analogue of the analysis presented in this article, showing that the space $S_{\rho_{n \mid h} \epsilon^{-3}, 3 / 2}(n)$ of cusp forms for $\Gamma_{0}(n)$ with weight $3 / 2$ and multiplier $\rho_{n \mid h} \epsilon^{-3}$ vanishes if and only if $(n, h)=\left(n_{g}, h_{g}\right)$ for some fixed-point-free $g \in M_{24}$. The fact that $S_{\rho_{n \mid h} \epsilon^{-3}, 3 / 2}(n)$ vanishes in case $(n, h)$ does arise from a fixed-point-free element of $M_{24}$ is established in CD12a].

Another natural direction to explore is the generalisation of our results to umbral moonshine CDH12. For $\ell$ an integer greater than 1 and $0<r<\ell$ define

$$
S_{r}^{(\ell)}=\sum_{m \in \mathbb{Z}}(2 \ell m+r) q^{(2 \ell m+r)^{2} / 4 \ell}
$$


Then $S_{1}^{(2)}$ recovers $\eta^{3}$ (cf. (1.2) $)$ and the vector-valued function $S^{(\ell)}=\left(S_{1}^{(\ell)}, \ldots, S_{\ell-1}^{(\ell)}\right.$ ) is a vector-valued cusp form for the modular group with weight $3 / 2$ and a certain matrix-valued multiplier $\sigma^{(\ell)}$. In [CDH12] a weak vector-valued mock modular form $H^{(\ell)}$ is constructed for each $\ell \in\{2,3,4,5,7,13\}$ whose shadow is proportional to $S^{(\ell)}$, and evidence is collected in support of a family of conjectures relating $H^{(\ell)}$ to a finite group $G^{(\ell)}$ in analogy to the relationship between $H$ and $M_{24}$ described above. In fact Mathieu moonshine may be regarded as the special case of umbral moonshine that $\ell=2$; we have $H^{(2)}=H$ and $G^{(2)}=M_{24}$. For a positive integer $n$ define $S_{\sigma^{(\ell)}, 3 / 2}(n)$ to be the space of $(\ell-1)$-vector-valued cusp forms of weight $3 / 2$ for $\Gamma_{0}(n)$ with multiplier system $\sigma^{(\ell)}$. Then $\operatorname{dim} S_{\sigma^{(\ell)}, 3 / 2}(n) \geq 1$ for all $n \geq 1$ since $S^{(\ell)} \in S_{\sigma^{(\ell)}, 3 / 2}(n)$. In light of the results of this paper, and upon inspection of the shadows described in [CDH12, it is tempting to suggest that the positive integers $n$ for which $\operatorname{dim} S_{\sigma^{(\ell)}, 3 / 2}(n)=1$ are exactly those such that there exists an element $g \in G^{(\ell)}$ of order $n$ with $\chi_{g}^{(\ell)}=\bar{\chi}_{g}^{(\ell)}>0$. This condition means that $g$ has fixed points but no anti-fixed points in the natural signed permutation representation of $G^{(\ell)}$. We refer to CDH12] for more details.

As is still the case for Ogg's observation on primes dividing the order of the monster, a conceptual explanation of the results of this article is not yet available. Nonetheless, we have seen in $\$ 1$ that both results may be motivated by the Rademacher sum construction of the Mathieu and monstrous McKay-Thompson series. As a result, it is tempting to envisage a class of algebraic structures whose twisted characters are given by Rademacher sums. The relation between this algebraic structure and vertex algebra should be a chiral counterpart to the relation between string theory on $A d S_{3}$ geometries and conformal field theory in two dimensions. See DF11, CD12a] for a more detailed discussion.

In the monstrous case this is a central implication of the results of [DF11, and the construction of a such an object with the monster acting as automorphisms would explain the genus zero property. This is because, as mentioned before, the relevant Rademacher sums have the required invariance if and only if the underlying discrete groups have genus zero. The existence of elements of prime order in the monster with associated discrete group of the form $\Gamma_{0}(p)^{+}$ then explains Ogg's observation. For the case of Mathieu moonshine the untwisted character is a Rademacher sum of weight $1 / 2$ with multiplier system $\psi=\epsilon^{-3}$ and we can expect the twisted characters to have shadows proportional to that of the untwisted character, which is to say, proportional to $\eta^{3}$ since $\operatorname{dim} S_{\epsilon^{3}, 3 / 2}(1)=1$ according to our results. As such, the construction of this algebraic structure would furnish an algebraic foundation for the conjectural characterisation of the discrete groups of Mathieu moonshine described above, and it would explain our analogue of Ogg's result: that the primes dividing the order of $M_{24}$ are exactly those for which $\operatorname{dim} S_{\epsilon^{3}, 3 / 2}(p)=1$. 


\section{A Special Functions}

For the exponential $x \mapsto x^{s}$ with $s$ a non-integer we employ the principal branch of the logarithm, so that

$$
x^{s}=|x|^{s} e^{\mathbf{i} s \theta}
$$

when $x=|x| e^{\mathrm{i} \theta}$ for $-\pi<\theta \leq \pi$.

The Dedekind eta function, denoted $\eta(\tau)$, is a holomorphic function on the upper half-plane defined by the infinite product

$$
\eta(\tau)=q^{1 / 24} \prod_{n>0}\left(1-q^{n}\right)
$$

where $q=\mathrm{e}(\tau)=e^{2 \pi \mathbf{i} \tau}$. It is a modular form of weight $1 / 2$ for the modular group $S L_{2}(\mathbb{Z})$ with multiplier $\epsilon: S L_{2}(\mathbb{Z}) \rightarrow \mathbb{C}$ so that

$$
\eta(\gamma \tau) \epsilon(\gamma) \mathrm{j}(\gamma, \tau)^{1 / 2}=\eta(\tau)
$$

for all $\gamma=\left(\begin{array}{ll}a & b \\ c & d\end{array}\right) \in S L_{2}(\mathbb{Z})$, where $\mathrm{j}(\gamma, \tau)=(c \tau+d)^{-1}$. The multiplier system $\epsilon$ may be described explicitly as

$$
\epsilon\left(\begin{array}{ll}
a & b \\
c & d
\end{array}\right)= \begin{cases}\mathrm{e}(-b / 24), & c=0, d=1 \\
\mathrm{e}(-(a+d) / 24 c+s(d, c) / 2+1 / 8), & c>0\end{cases}
$$

where $s(d, c)=\sum_{m=1}^{c-1}(d / c)((m d / c))$ and $((x))$ is 0 for $x \in \mathbb{Z}$ and $x-\lfloor x\rfloor-1 / 2$ otherwise. We can deduce the values $\epsilon(a, b, c, d)$ for $c<0$, or for $c=0$ and $d=-1$, by observing that $\epsilon(-\gamma)=\epsilon(\gamma) \mathrm{e}(1 / 4)$ for $\gamma \in S L_{2}(\mathbb{Z})$.

For an integer $a$ and an integer $n=\varepsilon \prod_{i=1}^{k} p_{i}^{e_{i}}$ with $\varepsilon= \pm 1$ and the $p_{i}$ mutually distinct primes, the Kronecker symbol $\left(\frac{a}{n}\right)$ is defined as follows. First we have the multiplication rule

$$
\left(\frac{a}{n}\right)=\left(\frac{a}{\varepsilon}\right) \prod_{i=1}^{k}\left(\frac{a}{p_{i}}\right)^{e_{i}}
$$


where the first factor is given by

$$
\left(\frac{a}{1}\right)=1, \quad\left(\frac{a}{-1}\right)= \begin{cases}-1 & a<0 \\ 1 & a \geq 0\end{cases}
$$

and for odd primes $p$ the Kronecker symbol $\left(\frac{p}{n}\right)$ is identical to the Legendre symbol

$$
\left(\frac{a}{p}\right)= \begin{cases}0 & p \mid a \\ 1 & a \text { is a quadratic residue } \bmod p \\ -1 & a \text { is not a quadratic residue } \bmod p\end{cases}
$$

and coincides with $\left(\frac{a}{p}\right)=a^{(p-1) / 2} \bmod p$. Finally, for $p=2$ we have

$$
\left(\frac{a}{2}\right)= \begin{cases}0 & 2 \mid a \\ 1 & a= \pm 1 \bmod 8 \\ -1 & a= \pm 3 \bmod 8\end{cases}
$$

\section{References}

$\left[\mathrm{CCN}^{+} 85\right]$ J.H. Conway, R.T. Curtis, S.P. Norton, R.A. Parker, and R.A. Wilson. Atlas of finite groups. Maximal subgroups and ordinary characters for simple groups. With comput. assist. from J. G. Thackray. Oxford: Clarendon Press, 1985.

[CD12a] Miranda C. N. Cheng and John F. R. Duncan. On Rademacher Sums, the Largest Mathieu Group, and the Holographic Modularity of Moonshine. Commun. Number Theory Phys., 6(3), 2012.

[CD12b] Miranda C. N. Cheng and John F. R. Duncan. The Largest Mathieu Group and (Mock) Automorphic Forms. January 2012.

[CD12c] Miranda C.N. Cheng and John F.R. Duncan. Rademacher Sums and Rademacher Series. 2012.

[CDH12] Miranda C. N. Cheng, John F. R. Duncan, and Jeffrey A. Harvey. Umbral Moonshine. 2012.

[Che10] Miranda C. N. Cheng. K3 Surfaces, $N=4$ Dyons, and the Mathieu Group $M_{24}$. May 2010. 
[CMS04] John Conway, John McKay, and Abdellah Sebbar. On the discrete groups of Moonshine. Proc. Amer. Math. Soc., 132:2233-2240, 2004.

[CN79] J. H. Conway and S. P. Norton. Monstrous Moonshine. Bull. London Math. Soc., 11:308 339, 1979.

[DF11] John F. R. Duncan and Igor B. Frenkel. Rademacher sums, moonshine and gravity. Commun. Number Theory Phys., 5(4):1-128, 2011.

[EH09] Tohru Eguchi and Kazuhiro Hikami. Superconformal Algebras and Mock Theta Functions 2. Rademacher Expansion for K3 Surface. Communications in Number Theory and Physics, 3,:531-554, April 2009.

[EH11] Tohru Eguchi and Kazuhiro Hikami. Note on Twisted Elliptic Genus of K3 Surface. Phys.Lett., B694:446-455, 2011.

[EOT11] Tohru Eguchi, Hirosi Ooguri, and Yuji Tachikawa. Notes on the K3 Surface and the Mathieu group $M_{24}$. Exper.Math., 20:91-96, 2011.

[Gan12] Terry Gannon. Much ado about Mathieu, November 2012.

[GHV10a] Matthias R. Gaberdiel, Stefan Hohenegger, and Roberto Volpato. Mathieu Moonshine in the elliptic genus of K3. JHEP, 1010:062, 2010.

[GHV10b] Matthias R. Gaberdiel, Stefan Hohenegger, and Roberto Volpato. Mathieu twining characters for K3. JHEP, 1009:058, 2010. 19 pages.

[Nie74] Douglas Niebur. Construction of automorphic forms and integrals. Trans. Amer. Math. Soc., 191:373-385, 1974.

[Ogg75] Andrew P. Ogg. Automorphismes de courbes modulaires. In Séminaire DelangePisotPoitou (16e année: 1974/75), Théorie des nombres, Fasc. 1, Exp. No. 7, page 8. Secrétariat Mathématique, Paris, 1975.

[Shi71] Goro Shimura. Introduction to the arithmetic theory of automorphic functions. Publications of the Mathematical Society of Japan, No. 11. Iwanami Shoten, Publishers, Tokyo, 1971. Kanô Memorial Lectures, No. 1.

[Ste07] William Stein. Modular forms, a computational approach, volume 79 of Graduate Studies in Mathematics. American Mathematical Society, Providence, RI, 2007. With an appendix by Paul E. Gunnells.

[Zag09] Don Zagier. Ramanujan's mock theta functions and their applications (after Zwegers and Ono-Bringmann). Astérisque, (326):Exp. No. 986, vii-viii, 143-164 (2010), 2009. Séminaire Bourbaki. Vol. 2007/2008. 
[Zwe02] Sander Zwegers. Mock Theta Functions. PhD thesis, Utrecht University, 2002. 